\title{
Aspects politiques et réglementaires
}

Oléagineux, Corps Gras, Lipides. Volume 10, Numéro 3, 187-90, Mai 2003, Colza : enjeux et nouvelles synergies de la recherche, ÉCONOMIE

Auteur(s) : Olivier de GASQUET, Directeur de la FOP, 12, avenue George V, 75008 Paris $<$ o.degasquetprolea.com> .

Author(s) : Olivier de GASQUET

Résumé : Depuis la mise en œuvre de la réforme de I'Agenda 2000 en 1999, la Commission européenne poursuit l'objectif implicite de libérer la production oléagineuse communautaire des contraintes résultant de I'accord de Blair House, auquel I'Europe avait dû souscrire en 1993 pour clore un long contentieux avec les Etats-Unis. II s'agit en fait pour elle de vider cet accord de sa substance par des mesures internes. Dans une première étape, ratifiée en 1999 par le Conseil européen de Berlin, elle a fait supprimer tout paiement spécifique aux oléagineux à usage alimentaire à compter de la récolte de 2002. Dans une deuxième étape, qui s'ouvre avec la réforme décidée le 26 juin 2003 à Luxembourg, elle généralise le découplage et met en place un paiement non spécifique aux cultures énergétiques. Mais cette stratégie trouve ses limites dans les distorsions de concurrence internes à la politique agricole commune, de même que dans celles liées à l'évolution de la politique agricole américaine. Sa réussite est donc largement conditionnée par les modalités d'application du " découplage " que prévoit la nouvelle réforme, tout autant que par la capacité de I’Union européenne à faire prévaloir ses intérêts au sein de l'organisation mondiale du commerce.

Summary : Since the launching in 1999 of the "Agenda 2000" reform, the European Commission has been struggling with the limitations the Blair House agreement put on the European oilseed production. While in 1993 the EU had to subscribe to this agreement in order to put an end to a longlasting dispute with the US, the Commission is now actually trying to make it irrelevant by way of domestic action. Their first step, which the Berlin European Council ratified in 1999, was the suppression of any specific payment to oilseeds for food purposes, starting with the 2002 crop. The second step, starting with the reform decided on June $26^{\text {th }} 2003$ in Luxembourg, is to extend "decoupling" and set up a non-specific payment to energy crops. But this strategy is hampered by the distortions of competition due to the common agricultural policy, as well as by those connected to American agricultural policy. Success hence largely hangs on precisely how "decoupling" is implemented according to the new reform \; the EU ability to have its interest prevail within the WTO will also be crucial.

Mots-clés : Agenda 2000, politique agricole commune, revue à mi-parcours, accord de Blair House, paiement spécifique, cultures énergétiques, organisation mondiale du commerce

Keywords : Agenda 2000, Common Agricultural Policy (CAP), Midterm Review (MTR), Blair House agreement, specific payment, energy crops, WTO 


\title{
ARTICLE
}

\author{
Auteur(s) : Olivier de GASQUET \\ Directeur de la FOP, 12, avenue George V, 75008 Paris \\ <o.degasquet@prolea.com>
}

Les oléagineux en général - et le colza en particulier - ont toujours occupé une place à part au sein de la politique agricole commune. Une remise en perspective est donc nécessaire si l'on veut comprendre l'enjeu de la nouvelle réforme de la politique agricole commune qui vient d'être décidée. Cela conduit d'une part à faire ressortir la stratégie " en creux » de l'Union européenne à son égard, d'autre part à examiner les paramètres internes et externes susceptibles de la favoriser ou de la contrarier.

\section{Une place à part au sein de la politique agricole commune}

Sans volonté politique, la production oléagineuse européenne n'aurait jamais pu se développer comme elle l'a fait. Au cours des quarante années qui séparent 1962 - date à laquelle l'Europe a consenti la suppression définitive de sa protection douanière sur les oléagineux - de l'année 2002 où il a été mis fin à toute politique spécifique en faveur de ce secteur, le défi permanent a été d'assurer le développement de la production dans un contexte réglementaire défavorable ${ }^{1}$.

${ }^{1}$ Pour plus de détails sur ces aspects historiques, voir Olivier de GASQUET (avril 2002), Comprendre notre agriculture et la PAC, 1-226, Editions VUIBERT, Paris.

\section{La concession douanière de 1962}

Lorsqu'au début des années 1960 la Communauté économique européenne a décidé de fonder la politique agricole commune sur des prélèvements (droits de douane variables), les Etats-Unis ont fait observer que ce mécanisme ne faisait pas partie de la panoplie des mesures autorisées par le GATT. Une négociation s'est donc engagée. Elle s'est conclue par une transaction au terme de laquelle les prélèvements européens ont été tolérés, contre l'engagement de la Communauté de renoncer définitivement à tout droit de douane sur les graines et tourteaux d'oléagineux. Cette concession a été consolidée par l'accord multilatéral du Dillon Round, signé le 7 mars 1962 à Genève.

L'organisation commune de marché des oléagineux, que la Communauté a mis en place le $1^{\mathrm{er}}$ juillet 1967, était donc totalement atypique. Contrairement à celles des céréales, du lait et de la viande bovine, qui étaient fondées sur la protection aux frontières et les prix élevés, la réglementation oléagineuse laissait la production européenne aux conditions du marché mondial. Mais elle prévoyait une compensation proportionnelle aux volumes produits, transitant par les triturateurs qui pouvaient ainsi rémunérer les producteurs à un prix supérieur à celui du marché.

\section{Les contentieux avec les Etats-Unis}

Dès la fin des années 1980 les Etats-Unis, constatant l'important développement des surfaces oléagineuses dans la Communauté, l'accusent d'avoir détourné l'accord de 1962. Certes, elle en respecte la lettre en n'appliquant pas de droit de douane. Mais l'aide qu'elle octroie à son industrie de transformation lui permet de payer un prix artificiellement élevé à ses producteurs, qui sont de 
cette manière incités à produire des oléagineux. Cela réduit d'autant les débouchés que les EtatsUnis étaient en droit d'attendre de l'accord de 1962.

En conséquence, les Etats-Unis demandent en 1988 la constitution d'un "panel » (instance d'arbitrage) dans le cadre du GATT. Celui-ci leur donne raison, et l'Europe modifie sa réglementation oléagineuse en octobre 1991. L'aide à la tonne dont bénéficiaient les triturateurs est remplacée par une aide à l'hectare directement octroyée aux producteurs. Cette première modification ne satisfait cependant pas les Etats-Unis qui demandent immédiatement la constitution d'un deuxième panel. Le conflit ne sera finalement résolu que dans le cadre d'un accord bilatéral global - portant à la fois sur l'ensemble du volet agricole de l'Uruguay Round et sur le contentieux oléagineux — conclu à Blair House le 20 novembre 1992.

\section{L'accord de Blair House et ses conséquences}

Le « mémorandum d'accord » que la Communauté approuve le 8 juin 1993 pour mettre en œuvre le compromis de Blair House a pour effet de limiter en Europe :

- les surfaces éligibles au paiement spécifique pour les oléagineux à 4,934 millions d'hectares au plus (cas d'un gel obligatoire de $10 \%$ ) pour l'Union européenne à quinze, dont 1,557 million d'hectares pour la France ;

- les cultures sur jachère industrielle par un seuil de transformation de 1 million de tonnes d'équivalent tourteau de soja.

C'est dans le cadre ainsi défini que la réglementation en vigueur jusqu'en 1999 prévoyait notamment :

- un système de pénalité dissuasif, destiné à faire respecter les surfaces éligibles au paiement spécifique (superficie maximale garantie) ;

- un paiement spécifique à l'hectare, variable en fonction de l'évolution du marché (avec franchise de $8 \%$ ) dont le montant de base appliqué aux rendement de référence entrant dans le calcul de ce paiement était de 94,24 écus par tonne pour les superficies comptabilisées dans la superficie maximale garantie ;

- le versement du paiement réservé à la jachère (68,83 écus par tonne de rendement de référence) pour les surfaces cultivées sur jachère industrielle.

Entre 1993 et 1999, le caractère fortement dissuasif du système de pénalité lié à l'application de la superficie maximale garantie faisait peser une contrainte forte sur le développement des surfaces oléagineuses à usage alimentaire. En revanche, la possibilité de cultiver les jachères a favorisé l'émergence - en France et en Allemagne notamment - d'une filière non alimentaire pourvoyeuse de nouveaux débouchés pour le colza. Mais le seuil de transformation de 1 million de tonnes d'équivalent tourteau de soja représentait une contrainte incompatible à terme avec les ambitions de l'Union européenne en matière de développement des énergies renouvelables. 


\section{Le colza et l'approvisionnement en protéines végétales de l'union européenne}

La concession douanière de 1962 a durablement placé l'industrie de l'alimentation animale européenne dans une situation de dépendance au regard de son approvisionnement en protéines végétales.

Pour schématiser, la ration des animaux d'élevage est composée d'une partie énergétique qui peut globalement être assurée par les céréales, et d'une partie protéique qui est apportée pour l'essentiel par les tourteaux d'oléagineux. En s'interdisant toute protection douanière sur les oléagineux lors de la mise en place de la politique agricole commune, l'Europe a considérablement limité sa capacité à encourager ce type de productions et a de facto ouvert son marché au tourteau de soja américain. Pour tenter de limiter cette dépendance, elle a néanmoins agi dans trois directions: - elle a introduit dès 1967 un régime spécifique de "paiement compensatoire " destiné à permettre aux triturateurs de combler l'écart entre le prix des oléagineux importés et le coût de production des graines en Europe; - elle a encouragé la production de graines protéagineuses (pois, féverole, lupin) après l'embargo que les Etats-Unis ont décidé en 1973 sur leurs exportations de tourteau de soja; - elle a développé l'utilisation des farines animales, en particulier des farines de viande, au prix d'une grave crise sanitaire (encéphalopathie spongiforme bovine) qui a fini par la conduire à suspendre durablement l'utilisation de ces farines (4 décembre 2000). Le taux d'approvisionnement de l'élevage européen en protéines a toujours été modeste. Néanmoins, le recours aux farines de viande lui permettait de se situer aux alentours de $33 \%$ en 1999-2000. L'interdiction de ces farines l'a fait retomber à $24 \%$ en 2001-2002. Le tableau ciaprès montre que ce taux est même limité à $23 \%$ en ce qui concerne les seules protéines végétales :

\begin{tabular}{|c|c|c|c|c|}
\hline \multicolumn{5}{|c|}{$\begin{array}{l}\text { Bilan européen (UE 15) des matières } \\
\text { (en tonnes équivalent tourteau de soja) }\end{array}$} \\
\hline & Production & & Consommation & $\begin{array}{ll}\text { Taux } & \text { de } \\
\text { couverture } & \end{array}$ \\
\hline Graines de colza & 3485 & Tourteaux de colza & 3841 & $91 \%$ \\
\hline Graines de tournesol & 1037 & $\begin{array}{l}\text { Tourteaux } \quad \text { de } \\
\text { tournesol }\end{array}$ & 2011 & $51 \%$ \\
\hline Graines de soja & 990 & Tourteaux de soja & 30776 & $3 \%$ \\
\hline Protéagineux & 1601 & Protéagineux & 2103 & $76 \%$ \\
\hline $\begin{array}{l}\text { Fourrages } \\
\text { déshydratés }\end{array}$ & 1503 & Fourrages déshydratés & 1428 & $105 \%$ \\
\hline
\end{tabular}




\begin{tabular}{|l|l|l|l|l|}
\hline Corn gluten feed & 842 & Corn gluten feed & 2685 & $31 \%$ \\
\hline Autres (1) & 708 & Autres (1) & 1441 & $49 \%$ \\
\hline Protéines végétales & $\mathbf{1 0 1 5 6}$ & Protéines végétales & $\mathbf{4 4 2 8 5}$ & $\mathbf{2 3 \%}$ \\
\hline Farine de poisson & 807 & Farine de poisson & 1467 & $55 \%$ \\
\hline Total & $\mathbf{1 0 9 6 3}$ & Total & $\mathbf{4 5 7 5 2}$ & $\mathbf{2 4 \%}$ \\
\hline
\end{tabular}

(1) Arachide, lin, coprah/palmiste, coton, tourteaux de germes de maïs. Source : FOP d'après Oil World

Le tableau permet également de mesurer la grande importance relative du colza comme source de protéines végétales d'origine européenne. A lui seul, il représente $34 \%$ des tonnages (tonnages de MRP, c'est-à-dire de matières riches en protéines, exprimés en équivalents tourteaux de soja) produits dans I'Union européenne. II devance largement les protéagineux (16\%), les fourrages déshydratés (15\%) et le tournesol (10\%). Au regard des quantités de MRP consommées, le tourteau de colza ne représente toutefois qu'un peu moins de $9 \%$, le tourteau de soja - provenant pour l'essentiel d'Amérique du Nord et du Sud - se taillant la part du lion avec pratiquement $70 \%$ du total $(30,8$ millions de tonnes de MRP, rapportées à une consommation totale de protéines végétales de 44,3 millions de tonnes de MRP).

Après le bannissement des farines de viande, un débat s'est instauré en Europe sur la nécessité de relancer la production de protéines végétales. Ne souhaitant prendre aucune mesure susceptible de contrarier le pari stratégique de l'Agenda 2000 en matière oléagineuse, et se situant dans la perspective de son futur projet de réforme (voir $\S 2$ ci-après), la Commission européenne a présenté le 19 mars 2001 un rapport concluant que "la meilleure source de protéines est le tourteau de soja » et que sa disponibilité sur le marché mondial " ne pose pas de problème ». Dans un premier temps au moins, cela signifie que l'accroissement de déficit consécutif à la suppression de l'utilisation des farines de viande doit être comblé par un accroissement des importations de tourteau de soja. Dès le mois de mars 2001, la Commission estimait ce surcroît d'importation à $+5 \%$. C'est effectivement l'ordre de grandeur de l'évolution observée depuis lors.

\section{Le pari stratégique de l'Agenda 2000}

Avec l'Agenda 2000 - qui couvre la période 2000-2006 - la Commission européenne poursuit l'objectif implicite de libérer la production oléagineuse des contraintes résultant de l'accord de Blair House, en faisant perdre à celui-ci son objet :

- la première étape, ratifiée en 1999 par le Conseil européen de Berlin, a abouti à supprimer tout paiement à l'hectare spécifique aux oléagineux à compter de 2002 ;

- la deuxième, envisagée dès juillet 2002 au titre de la « revue à mi-parcours » de l'Agenda 2000, en cours de discussion au sein du Conseil des ministres européens de l'Agriculture, consistait à 
généraliser le découplage et à substituer à la jachère industrielle un paiement non spécifique aux cultures énergétiques.

\section{La suppression de tout paiement spécifique pour les oléagineux}

Le Conseil européen de Berlin des 24 et 25 mars 1999 a programmé un démantèlement des paiements spécifiques aux oléagineux sur trois ans :

- suppression de la variabilité du paiement dès la récolte de 2000 ;

- alignement sur le montant du paiement à l'hectare pour les céréales (63 euros par tonne de rendement de référence) à compter de la récolte de 2002.

Alors que le différentiel de paiement à l'hectare au sein des cultures arables s'établissait pratiquement à 40 euros par tonne de rendement de référence en 1999 (94,24 euros par tonne pour les oléagineux, contre 54,34 pour les céréales), il a été réduit à partir de 2000 pour être supprimé en 2002. Même s'il est vrai qu'au cours de cette période le prix d'intervention des céréales a été abaissé de $15 \%$, il en est résulté une incontestable perte de compétitivité relative au sein des cultures arables, qui explique que les surfaces européennes d'oléagineux en général - et de colza en particulier - aient baissé de $15 \%$ en trois ans.

Dans la mesure où l'Agenda 2000 prévoyait une clause de rendez-vous stipulant que la Commission " devra suivre de près l'évolution du marché oléagineux et présenter un rapport dans un délai de deux ans à compter de l'application du nouveau régime " et qu'au besoin "ce rapport sera assorti de propositions appropriées si le potentiel de production devait se détériorer sérieusement ", la question était notamment de savoir si la "revue à mi-parcours " serait l'occasion de revenir d'une façon ou d'une autre sur ces dispositions. Or la Commission européenne, jugeant en juillet 2002 qu'il n'y avait "aucune détérioration importante du potentiel de production européen (...) dans un avenir prévisible ", n'a pas jugé " nécessaire, à ce stade, d'établir un nouveau rapport $»^{2}$.

En vérité, la Commission européenne ne souhaitait pas remettre en question, au travers de la future réforme qu'elle entendait proposer au titre de la "revue à mi-parcours » de l'Agenda 2000, la stratégie qu'elle avait réussi à faire avaliser par le Conseil européen en 1999. Ayant l'intention de préconiser une nouvelle baisse du prix d'intervention des céréales et un renforcement $d u$ " découplage " des soutiens aux cultures arables, elle considérait en effet que cela suffirait à améliorer la compétitivité relative des oléagineux. Le compromis auquel les ministres de l'Agriculture sont parvenus le 26 juin 2003 ne lui permet toutefois d'atteindre son objectif que partiellement, dans la mesure où le prix réglementé des céréales n'est finalement modifié qu'au travers de la réduction de moitié des majorations mensuelles.

${ }^{2}$ Commission des Communautés européennes (Juillet 2002), Communication de la Commission au Conseil et au Parlement européen, Révision à mi-parcours de la politique agricole commune, 3-42.

\section{La proposition de "crédit carbone " pour les cultures énergétiques}

L'utilisation accrue de biocarburants dans les transports fait partie des mesures que I'Union européenne entend prendre pour respecter ses engagements en matière de changement climatique (protocole de Kyoto notamment), ainsi que pour assurer une sécurité d'approvisionnement 
respectueuse de l'environnement et promouvoir les sources d'énergie renouvelable. Une directive adoptée en avril 2003 fixe à cet égard un objectif de production atteignant $2 \%$ de la quantité totale d'essence et de gazole utilisée dans les transports d'ici à la fin de 2005 , et 5,75\% d'ici à la fin de $2010^{3}$.

Dans le cadre de la " revue à mi-parcours » de l'Agenda 2000, la Commission européenne proposait à cet effet de remplacer les dispositions actuelles en matière de jachère industrielle - qui, en ce qui concerne les oléagineux, sont soumises au seuil de transformation de 1 million de tonnes d'équivalent tourteau de soja - par un paiement non spécifique en faveur de toutes les cultures énergétiques ayant vocation à se substituer au dioxyde de carbone. Dans le projet de règlement soumis aux ministres de l'Agriculture, ce nouveau paiement - baptisé "crédit carbone " - était proposé à 45 euros par hectare, dans la limite d'une superficie maximale garantie de 1,5 million d'hectares.

Dès les premières discussions concernant ce point particulier du projet de la Commission, il est toutefois apparu que si - à moyen terme - une telle orientation pouvait effectivement libérer la production d'oléagineux de la contrainte de Blair House liée à la culture sur jachère, le passage brutal d'un système à un autre était susceptible de déstabiliser gravement les filières existantes. A cela s'ajoutait le fait que les montants proposés par la Commission semblaient à première vue notoirement insuffisants ${ }^{4}$. La Commission européenne a donc admis sans grande difficulté qu'un tel dispositif devrait être introduit de façon beaucoup plus progressive, avec le maintien de la possibilité de cultiver les jachères.

${ }^{3}$ Directive du Parlement européen et du Conseil visant à promouvoir l'utilisation de biocarburants ou autres carburants renouvelables dans les transports.

${ }^{4}$ A titre indicatif, la délégation française avait défendu le principe d'un montant de 100 euros par hectare en 1999, lorsque cette idée avait été émise pour la première fois dans le cadre de la négociation de l'Agenda 2000. II est clair par ailleurs qu'en l'absence du maintien de la possibilité de cultiver les jachères à des fins non alimentaires, une SMG de 1,5 million d'hectares n'aurait pas été compatible avec les objectifs de la directive sur les biocarburants, surtout dans la perspective d'une Union européenne élargie.

\section{Les paramètres qui conditionnent l'avenir}

La réforme du 26 juin 2003 et son application à l'Union européenne élargie, ainsi que l'évolution des négociations au sein de l'organisation mondiale du commerce - y compris en ce qui concerne la question des organismes génétiquement modifiés - constituent les facteurs internes et externes qui influenceront la production de colza au cours des années à venir.

\section{Facteurs internes}

Si l'on se situe dans une perspective longue, les décisions qui viennent d'être prises s'inscrivent dans une grande continuité. Depuis 1992, I'Union européenne a entrepris, sous l'impulsion de la Commission, de " découpler » les soutiens à l'agriculture en transformant par étapes les paiements aux produits en paiements aux facteurs de production (hectare ou animal), l'objectif ultime étant le paiement à l'exploitation. 
Force est de constater qu'en la matière le secteur des oléagineux avait pris une longueur d'avance, le paiement étant déjà intégralement versé sur le critère de facteur de production. Le renforcement du découplage le touche donc moins que les autres secteurs, et peut même contribuer à rétablir les équilibres en sa faveur, toutes choses égales par ailleurs.

Dans la perspective d'une disparition progressive des moyens d'orientation des productions mis en place dans les années 1960, il est clair que l'évolution de la réglementation sur le développement rural (aussi appelée "deuxième pilier» de la politique agricole commune) peut permettre de retrouver des leviers d'une autre nature, au travers notamment de son volet agroenvironnemental. L'expérience conduite depuis 2002 en France pour promouvoir une mesure de " diversification des cultures dans l'assolement " laisse penser qu'une production comme le colza pourrait, avec d'autres, en recueillir là aussi les effets indirects. Mais cela reste bien entendu subordonné aux choix politiques et budgétaires qui devront être opérés à cet égard, tant au niveau européen que national, puisque ces mesures sont cofinancées. On peut relever à ce sujet que la décision prise le 26 juin 2003 de porter le taux de participation communautaire au mesures agroenvironnementales de $50 \%$ à $60 \%$ est susceptible d'en faciliter le financement, notamment en France.

\section{Facteurs externes}

L'élargissement de l'Union à dix nouveaux membres à partir de 2004, ainsi que les négociations au sein de l'organisation mondiale du commerce - dont la conclusion est normalement prévue pour le $1^{\text {er }}$ janvier 2005 au plus tard - constituent le cadre dans lequel évoluera la politique agricole commune cours des prochaines années. Concernant les oléagineux, l'évolution divergente de la politique agricole américaine est un sujet de préoccupation récurrent en Europe depuis plusieurs années. Le FAIR Act (loi agricole américaine s'appliquant à la période 1996-2002) a en effet rendu le soja éligible aux paiements à la surface (aides de "flexibilité ") alors qu'il ne l'était pas auparavant, ce qui a incité au développement des surfaces. A cela s'ajoute l'effet des «loan deficiency payments » sur les prix de marché.

En effet, depuis la baisse des prix mondiaux qui sont passés en dessous des "loan rates " en 1998/99, les surfaces américaines de soja ont continué à progresser (+ $25 \%$ en cinq ans), bien que le prix du soja soit tombé à son plus bas niveau depuis 25 ans, et que les superficies diminuent dans les autres pays. En injectant massivement des paiements compensatoires ("loan deficiency payments ») proportionnels aux volumes produits pour neutraliser les effets de cette baisse auprès des producteurs américains, les Etats-Unis ont aggravé l'effondrement des cours, atteignant le double objectif de conforter leur propre production d'oléagineux en décourageant celle de leurs concurrents. Ces évolutions sont contraires aux dispositions du volet agricole de l'accord commercial de Marrakech (15 avril 1994).

C'est la raison pour laquelle l'alliance européenne pour les oléagineux (EOA $)^{\frac{5}{5}}$ vient de déposer une plainte auprès de la Commission européenne, dans le cadre de la procédure dite de « Règlement des Obstacles au Commerce " (ROC). Cette démarche vise à amener la Commission à engager une "procédure en règlement des différends" contre la politique oléagineuse américaine devant I'organisation mondiale du commerce. Ce test de cohérence et de vérité permettra de vérifier si l'Europe a les moyens de faire respecter aux autres les disciplines qu'elle s'impose à elle-même. Le 
pari stratégique consistant à opérer la reconquête des protéines végétales au travers d'un découplage renforcé du soutien aux cultures arables n'est en effet tenable que si les partenaires mondiaux de l'Union européenne mènent des politiques allant dans le même sens.

${ }^{5}$ Présidée par Xavier Beulin (FOP, France), I'EOA regroupe les organisations représentatives du secteur des oléoprotéagineux des principaux pays producteurs de l'Union européenne (aujourd'hui, I'Allemagne, la France, le Royaume-Uni, l'Espagne, la Suède, la Belgique). 